\title{
Reducing the critical switching current in nanoscale spin valves
}

\author{
Jan Manschot ${ }^{\mathrm{a})}$ \\ Kavli Institute of Nanoscience, Delft University of Technology, 2628 CJ Delft, The Netherlands and \\ Department of Physics, Norwegian University of Science and Technology, N-7491 Trondheim, Norway \\ Arne Brataas \\ Department of Physics, Norwegian University of Science and Technology, N-7491 Trondheim, Norway
}

Gerrit E. W. Bauer

Kavli Institute of Nanoscience, Delft University of Technology, 2628 CJ Delft, The Netherlands

(Received 5 April 2004; accepted 3 August 2004)

\begin{abstract}
The current induced magnetization reversal in nanoscale spin valves is a potential alternative to magnetic field switching in magnetic memories. We show that the critical switching current can be decreased by an order of magnitude by strategically distributing the resistances in the magnetically active region of the spin valve. In addition, we simulate full switching curves and predict a new precessional state. (C) 2004 American Institute of Physics. [DOI: 10.1063/1.1801169]
\end{abstract}

The prediction that a spin-polarized current can excite and reverse a magnetization ${ }^{1,2}$ has been amply confirmed by recent experiments. ${ }^{3,4}$ The current-induced magnetization dynamics is interesting as an efficient mechanism to write information into magnetic random access memories as well as to generate microwaves. ${ }^{5}$ Unfortunately, the critical currents for magnetization reversal are still unattractively high. ${ }^{6}$ In this letter, we apply a previously developed microscopic formalism ${ }^{7}$ to understand the critical current in spin valves quantitatively and propose a strategy to reduce it by up to an order of magnitude. We also solve the micromagnetic equations with accurate angle-dependent magnetization torque and spin-pumping ${ }^{8}$ terms and predict switching to a precessional state.

We will first consider a generic $\mathrm{F}$ (erromagnetic) $\mid \mathrm{N}$ (ormal) $\mid \mathrm{F}$ spin valve biased by a voltage difference $V$. The two ferromagnetic reservoirs are assumed to be monodomain; the magnetizations differ by an angle $\theta$. Charge and spin currents excited by an applied bias can be calculated accurately by magneto-electronic circuit theory ${ }^{7}$ with parameters determined from first-principle calculations ${ }^{9}$ that agree well with experimental data. ${ }^{10}$ To this end we dissect the pillar into three nodes (the reservoirs and the normal metal) connected by two, not necessarily identical resistive elements $G_{L}$ and $G_{R}$. Each of them is characterized by the conductance $g=g^{\uparrow \uparrow}+g^{\downarrow \downarrow}$, the polarization $p=\left(g^{\uparrow \uparrow}-g^{\downarrow \downarrow}\right) / g$ and the normalized mixing conductance $\eta=2 g^{\uparrow \downarrow} / g$. $g^{\uparrow \uparrow}$ and $g \downarrow \downarrow$ are, respectively, the conductances for electrons with spin parallel and antiparallel to the magnetization and $g^{\uparrow \downarrow}$ is the material parameter that governs the magnetization torque. The magnetically active region includes layers of thickness up to the spin-flip diffusion lengths from the interfaces. Any resistance outside this region is parasitic and not considered here. The conductances are effective parameters determined by the resistance of the ferromagnetic and normal metal bulk, that of the interfaces to the normal metal and the resistance of an eventual outer normal metal that fits into the magnetically active region. For simplicity we disregard the bulk resistance of the normal metal island and the imaginary

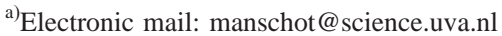

part of $g^{\uparrow \downarrow}$ (for metallic interfaces smaller than $10 \%$ of the real part $\left.{ }^{9,11}\right)$.

The transverse component of the spin current is absorbed in the ferromagnet ${ }^{11}$ and the associated spin-transfer torque can excite the magnetization. ${ }^{1,2}$ Circuit theory has been used to derive analytic expressions for the torques in a symmetrical spin valve as function of the angle $\theta$ between the magnetization directions. ${ }^{10}$ However, the spin-transfer torque depends strongly on the resistance distribution, even its sign may change with asymmetry. ${ }^{12,13}$ In this letter we propose to engineer the spin valve resistance distribution in order to minimize the critical current for magnetization reversal from the parallel to the antiparallel configuration (the opposite process is much less sensitive to this asymmetry). Expanding the spin-transfer torque from circuit theory to first order in $\theta$, the normalized torque $i_{s}=\mid \boldsymbol{m} \times\left(\boldsymbol{I}_{s} \times \boldsymbol{m}\right) / I_{c}$ on the left magnetization reads

$$
\begin{aligned}
\left.i_{s}(\theta)\right|_{\theta \approx 0} & \\
= & \frac{\hbar}{2 e}\left(\frac{g_{L} \eta_{L}}{g_{L} \eta_{L}+g_{R} \eta_{R}}\right) \\
& \times \frac{g_{R} \eta_{R}\left(p_{R}-p_{L}\right)+g_{L} p_{R}\left(1-p_{L}^{2}\right)+g_{R} p_{L}\left(1-p_{R}^{2}\right)}{g_{L}\left(1-p_{L}^{2}\right)+g_{R}\left(1-p_{R}^{2}\right)} \theta .
\end{aligned}
$$

In an asymmetric structure, $p_{R}-p_{L} \neq 0$, the slope of the spin torque can be considerably enhanced compared to the torque in a symmetric structure $i_{s}=(\hbar / 2 e)(p / 2) \theta$.

We investigate a realistic (but nonunique) model for the asymmetry by extending the layer sequence from $\mathrm{F}|\mathrm{N}| \mathrm{F}$ to $\mathrm{N}_{1}|\mathrm{~F}| \mathrm{N}|\mathrm{F}| \mathrm{N}_{2}$. We take here the four $\mathrm{N} \mid \mathrm{F}$ interfaces to be equal and assume that the ferromagnets are thin enough that the bulk contribution is negligibly small. Numerical results require values for the interface resistances that have been measured accurately in the current-perpendicular-to-plane geometry. ${ }^{14,15}$ We adopt here $\mathrm{Co} / \mathrm{Cu}$ interfaces with crosssection $1.26 \times 10^{4} \mathrm{~nm}^{2}$, whence $1 / G=0.0183 \Omega, p=0.75$, and $\eta=0.38 .^{9,16}$ The asymmetry is modeled by the normal metal sandwich outside the symmetric $\mathrm{F}|\mathrm{N}| \mathrm{F}$ structure. The conductivity of the resistive element connecting the left (right) reservoir to the adjacent normal metal layer is $G_{1}\left(G_{2}\right)$. The asymmetry is expressed by varying the values for $G_{1}$ 
TABLE I. The slope of the spin torque at $\theta \approx 0$, the increase of the damping by spin pumping, and the critical current for different asymmetrical configurations of the investigated finite element system.

\begin{tabular}{lccc}
\hline \hline$G_{1}: G_{2}$ & $k$ & $\Delta \alpha$ & $I_{c, c}$ \\
\hline $1: \infty$ & 0.565 & 0.0054 & $0.55 \mathrm{~mA}$ \\
$1: 1$ & 0.117 & 0.0062 & $2.86 \mathrm{~mA}$ \\
$\infty: 1$ & -0.331 & 0.0131 & $-1.59 \mathrm{~mA}$ \\
\hline \hline
\end{tabular}

and $G_{2}$ for constant series resistance $1 / G_{1}+1 / G_{2}=0.37 \Omega$. The total collinear pillar resistances can now be calculated by the two current model to be 0.505 and $0.534 \Omega$, which are typical values for recently fabricated nanopillars. ${ }^{3}$ We assume that the right magnetic layer is magnetically hard and is treated as static "polarizer."

The effective conductance parameters, $\left(\hat{g}_{L}, \hat{p}_{L}\right.$, and $\left.\hat{\eta}_{L}\right)$ for the left-hand side of the pillar consisting of the ferromagnet and the outer normal metal can be calculated in terms of the normal metal conductance $g_{1}$ as

$$
\begin{aligned}
& \hat{g}_{L}=\frac{1}{2}\left(\frac{g_{1} g^{\uparrow \uparrow}}{g_{1}+g^{\uparrow \uparrow}}+\frac{g_{1} g^{\downarrow \downarrow}}{g_{1}+g^{\downarrow \downarrow}}\right), \quad \hat{\eta}_{L}=\frac{2 g^{\uparrow \downarrow}}{\hat{g}_{L}}, \\
& \hat{p}_{L}=\frac{g_{1}^{2}\left(g^{\uparrow \uparrow}-g^{\downarrow \downarrow}\right)}{2\left(g_{1}+g^{\uparrow \uparrow}\right)\left(g_{1}+g^{\downarrow \downarrow}\right) \hat{g}_{L}} .
\end{aligned}
$$

$g_{1}$ should be replaced by $g_{2}$ to obtain expressions for the right-hand side. We parametrize Eq. (1) as $i_{s}=(\hbar / 2 e) k \theta$, where numerical results for the torque parameter $k$ are given in Table I for different distributions of the resistance over $G_{1}$ and $G_{2}$. The dependence of the torque on $G_{1}: G_{2}$ can be understood simply in terms of the spin accumulation in the parallel configuration. In contrast to a symmetric structure, it does not vanish in asymmetric valves and can have either sign. ${ }^{12}$ The additional spin accumulation that is excited when $\theta$ becomes finite increases an antiparallel accumulation, and thus the torque, when the extra resistance is on the left-hand side. In the opposite case, the initially positive spin accumulation is canceled at a certain angle at which the angular magnetoresistance is minimal and the torque vanishes. ${ }^{12}$

For our specific example the spin-transfer torque on the left magnetization is enhanced by a factor of 5 when all normal resistance resides on the side of the left magnetic layer. The torque can thus be maximized by placing a material with a small spin flip length (e.g., platinum) adjacent to the right magnetic layer, as well as a material with a large spin flip length (e.g., copper) to the left layer. The magnitude of the torque is enhanced as well when all resistance is placed on the other side, but its sign is changed. As shown in the following, in this configuration a reversed current induces switching to a finite angle.

The magnetization dynamics is described by an extended form of the Landau-Lifshitz-Gilbert equation. ${ }^{17,18}$ As in previous simulations ${ }^{19,20}$ we adopt a single-domain model, but we take into account accurate angle-dependent magnetization torques ${ }^{12}$ as well as the "dynamic stiffness" 8 due to spin pumping. ${ }^{21}$ We take the layers to be in the $y-z$ plane and the $x$ axis in the current direction. A uniaxial effective field, $\boldsymbol{B}_{\text {eff }}$, and the fixed magnetization are chosen parallel to the $z$ axis. Disregarding dipole and exchange coupling between the magnetic layers, both magnetizations in the ground state point along the external field. Analytic estimates of the criti- cal current are obtained here by focusing on the instability point, at which the current-induced torque exactly equals the damping torque $D(\theta)$. In the presence of in-plane fields, the critical current for complete switching does not necessarily agree with the instability point. ${ }^{22}$ We disregard this complication as well as temperature induced fluctuations of the magnetizations since they do not interfere with the effect of the distributed resistance.

The maximal viscous damping reads to lowest order in small angles in $\theta$ from the parallel configuration:

$$
\left.D(\theta)\right|_{\theta \approx 0}=\alpha M_{1}\left|\boldsymbol{B}_{\text {eff }}\right| \theta,
$$

where $\alpha$ is the Gilbert damping parameter and $M_{1}$ is the magnitude of the left magnetic moment. We obtain numerical results for $M_{1}$ with the sample cross section defined above, a thickness of $3 \mathrm{~nm}$ and a saturation magnetization $M_{s}=1.19$ $\times 10^{6} \mathrm{~A} \mathrm{~m}^{-1}$. The critical current $I_{c, c}$ is then given by

$$
I_{c, c}=\left.\frac{D(\theta)}{i_{s}(\theta)}\right|_{\theta \approx 0}=\frac{2 e\left|\boldsymbol{B}_{\mathrm{eff}}\right| M_{1}}{\hbar} \frac{\alpha}{k} .
$$

The total Gilbert damping parameter $\alpha$ consists of $\alpha_{0}$ $=0.006$, the bulk Gilbert damping parameter and $\Delta \alpha(\theta)$ originating from the dynamic stiffness. ${ }^{8,21}$ We find for $\left.\Delta \alpha\right|_{\theta \approx 0}$ in the limit that the spin currents are efficiently dissipated

$$
\left.\Delta \alpha\right|_{\theta \approx 0}=\frac{\gamma \hbar}{8 \pi M_{1}}\left(\frac{2 g_{1} g^{\uparrow \downarrow}}{g_{1}+2 g^{\uparrow \downarrow}}+g^{\uparrow \downarrow}\right) .
$$

The first term in parentheses is the conductance for a transverse spin current from the (left) ferromagnet to the left reservoir. The transverse spins escaping to the right are dissipated in the ferromagnet when $\theta \approx 0$, the conductance for these spins is thus $\eta g / 2$. The distribution of the resistance over $G_{1}$ and $G_{2}$ is thus of importance as well for the magnitude of the Gilbert damping. Decreasing $g_{1}$ decreases the damping parameter and thus the critical current. In Table I, the excess damping $\Delta \alpha$ is given for several resistance distributions.

The critical currents $I_{c, c}$ can now be calculated assuming an effective field of $\left|\boldsymbol{B}_{\text {eff }}\right|=0.2 \mathrm{~T}$. We observe that moving the resistance to the side of the switching layer decreases the critical current in two ways, by decreasing the excess damping and increasing the torque. For our specific model structure the critical current is more than five times smaller in the most asymmetric compared to the symmetric pillar. When all resistance resides on the right-hand side of the pillar, the lowest critical current is achieved by an opposite bias. Not only the torque, as shown above, but also the damping is then increased. Measured critical currents can be modeled generally well with our model (within 10\%) when anisotropy fields are included and, in some samples, the dynamics of the polarizer.

A Pt layer insertion (with very short spin-flip diffusion length) close to the switching layer as fabricated by Kiselev et $a l .{ }^{4}$ reduces the magnetically active resistance on the left side. However, this paper does not report an inverse switching as predicted here. In fact, our calculated critical currents agree best with the experimental ones for a symmetric structure without any resistance outside the magnetic layers of equal thickness. This ambiguity might be caused by the limilicense or copyright; see http://apl.aip.org/about/rights_and_permissions 


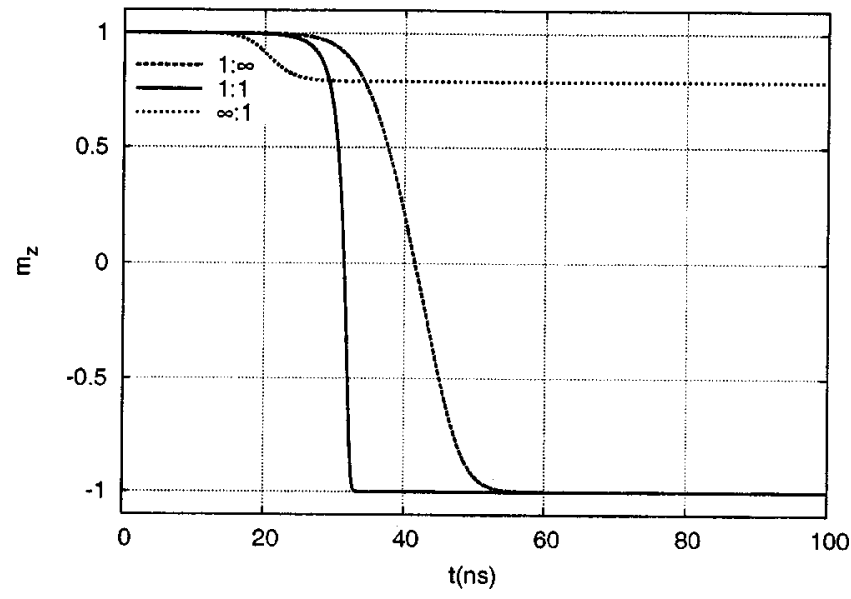

FIG. 1. $m_{z}$ as function of time for switching in magnetic multilayers with different resistance distributions. The legend denotes the ratio of $G_{1}: G_{2}$.

tation of our one-dimensional model to accurately describe the three-dimensional magnetic polarizing contact in the device of Kiselev et al.

Finally, we present numerical simulations of complete switching curves. A small initial torque is created by starting at $\theta_{0}=0.001$. In Fig. 1 we present the switching curves $m_{z}(t)$ of the left magnetization for three resistance distributions. $m_{z}(t)$ is the $z$ component of the unit vector $\boldsymbol{M}_{1} /\left|\boldsymbol{M}_{1}\right|$. All curves are calculated with $c=I_{c} / I_{c, c}=1.5$. We observe that for $\infty: 1$ the magnetization switches to an angle between $\theta$ and $\pi$ when the current bias is opposite. The origin of this state clearly differs from previously reported precessional states, ${ }^{4,23}$ which required that the applied field is not parallel to the polarizing (fixed) magnetization. It is a direct consequence of the sign change in the torque as function of the angle. ${ }^{12}$ The switching curves for small deviations from 1 of the different configurations can be approximated by (1 $\left.-m_{z}(t)\right) /\left(1-m_{z}(0)\right)=\exp \left(2 \alpha \gamma\left|\boldsymbol{B}_{\text {eff }}\right|(c-1) t\right)$. A smaller damping parameter thus increases the switching time but decreases the critical current. The angular dependence of the spin torque affects the whole switching curve; the torque resembles a sine function for $1: \infty$, whereas the symmetrical case is closer to Slonczewski's expression. ${ }^{1}$

Based on analytic expressions for the spin torque and spin pumping near $\theta=0$ in magnetic multilayers we conclude that the critical current for magnetization reversal in nanoscale spin valves can be reduced by up to an order of mag- nitude by engineering the resistance distribution in the magnetically active region. The spin torque changes sign for specific asymmetries giving rise to a new precessional state. After submission of this letter Jiang et $a l^{24}$ reported a strongly reduced switching current by modifying the resistance distribution in the nanopillar by a $\mathrm{Ru}$ insertion.

The authors would like to thank A. D. Kent for fruitful discussions. This work was financially supported by FOM, the Research Council of Norway, NANOMAT Grants No. 158518/431 and 158547/431, and the NEDO joint research program "Nano-Scale Magneto-electronics."

${ }^{1}$ J. C. Slonczewski, J. Magn. Magn. Mater. 159, L1 (1996).

${ }^{2}$ L. Berger, Phys. Rev. B 54, 9353 (1996).

${ }^{3}$ B. Oezyilmaz, A. D. Kent, D. Monsma, J. Z. Sun, M. J. Rooks, and R. H. Koch, Phys. Rev. Lett. 91, 67203 (2003).

${ }^{4}$ S. I. Kiselev, J. C. Sankey, I. N. Krivorotov, N. C. Emley, R. J. Schoelkopf, R. A. Buhrman, and D. C. Ralph, Nature (London) 425, 380 (2003).

${ }^{5}$ W. H. Rippard, M. R. Pufall, S. Kaka, S. E. Russek, and T. J. Silva, Phys. Rev. Lett. 92, 027201 (2004).

${ }^{6}$ J. Z. Sun, Nature (London) 425, 359 (2003).

${ }^{7}$ A. Brataas, Y. V. Nazarov, and G. E. W. Bauer, Phys. Rev. Lett. 84, 2481 (2000).

${ }^{8}$ Y. Tserkovnyak, A. Brataas, and G. E. W. Bauer, Phys. Rev. Lett. 88, 117601 (2002).

${ }^{9}$ K. Xia, P. J. Kelly, G. E. W. Bauer, A. Brataas, and I. Turek, Phys. Rev. B 65, 220401 (2002).

${ }^{10}$ G. E. W. Bauer, Y. Tserkovnyak, D. Huertas-Hernando, and A. Brataas, Phys. Rev. B 67, 94421 (2003).

${ }^{11}$ M. D. Stiles and A. Zangwill, Phys. Rev. B 66, 14407 (2002).

${ }^{12}$ J. Manschot, A. Brataas, and G. E. W. Bauer, Phys. Rev. B 69, 092407 (2004).

${ }^{13}$ A. A. Kovalev, A. Brataas, and G. E. W. Bauer, Phys. Rev. B 66, 224424 (2002).

${ }^{14}$ W. P. Pratt, Jr., S. F. Lee, J. M. Slaughter, R. Loloee, P. A. Schroeder, and J. Bass, Phys. Rev. Lett. 66, 3060 (1991).

${ }^{15}$ M. A. M. Gijs, S. K. J. Lenczowski, and J. B. Giesbers, Phys. Rev. Lett. 70, 3343 (1993).

${ }^{16}$ Q. Yang, P. Holody, R. Loloee, L. L. Henry, W. P. Pratt, Jr., P. A. Schroeder, and J. Bass, Phys. Rev. B 51, 3226 (1995).

${ }^{17}$ E. M. Lifshitz and L. P. Pitaevskii, Statistical Physics, Part 2 (Pergamon, New York, 1980).

${ }^{18}$ T. L. Gilbert, Phys. Rev. 100, 1243 (1955).

${ }^{19}$ J. Z. Sun, Phys. Rev. B 62, 570 (2000).

${ }^{20} \mathrm{Z}$. Li and S. Zhang, Phys. Rev. B 69, 134416 (2004).

${ }^{21}$ Y. Tserkovnyak, A. Brataas, and G. E. W. Bauer, Phys. Rev. B 67, 140404 (2003).

${ }^{22} \mathrm{~T}$. Valet (unpublished).

${ }^{23}$ Z. Li and S. Zhang, Phys. Rev. B 68, 024404 (2003).

${ }^{24}$ Y. Jiang, S. Abe, T. Ochiai, T. Nozaki, A. Hirohata, N. Tezuka, and K. Inomata, Phys. Rev. Lett. 92, 167204 (2004). 